\title{
Grandparents and Grandparenting in Iran: An Approach to Environmental Gerontology
}

\author{
Mohammad Taghi Sheykhi* \\ Department of Social Science, Alzahra University, Tehran \\ *Corresponding author: Mohammad Taghi Sheykhi, Professor of Sociology, Department of Social Science, Alzahra University, Tehran, Iran \\ Submission: 眥 January 16, 2018; Published: 眥 May 11, 2018
}

\begin{abstract}
As a branch of the human sciences, gerontology tries to depict the facts about old age which includes grandparents as well. But, the approach of gerontology sometimes look at the meaning from the outside. According to one widely shared view, the agenda for social gerontology should be to promote better social integration of the aged/grandparents by means of domestic activities, grand parenting and participatory roles in all kinds. Whether through work, leisure, or attendance at religious services, the aim of social integration is for people to remain engaged throughout life. This new and increasing age group that has its own life style needs special care, nursing, housing, security and economy. While grandparents are increasing, on the other hand, social structure is ever changing in Iran - families are contracting, girls are working, or staying outside home and so forth. The emerging process is having serious impacts on the elderly or grandparents. The paper illustrates the life conditions of grandparents; their needs, their quality of life and how they interact. As the phenomenon is almost new to Iran, the country does not have experience enough to put up with it, as it happens in the developed world. Method of research used in the current survey is a combined method of theoretical and empirical paradigms. As the number is increasing, the population needs more investment from different perspectives [1].
\end{abstract}

Keywords: Aging people; Gerontology; Grandparents; Quality of Life; Social integration

\section{Introduction}

As aging and longevity are gradually increasing in Iran, the present study aims to find out the health and socio-demographic conditions of those people whom we call grandparents in this paper. Though there are very few sources and scientific documents on grandparents in Iran, the author is trying to create views and literature on the topic by collecting data and using relevant materials through referring to foreign resources. While the concept of "Third Age" was not very popular in the past, it has found its deep meaning during the twentieth century, in which industry and medicine have created miracles - leading to aging and increase in the number of grandparents.

While in the past grandparents were very influential over the youth, social structure of the family was such that, almost every decision-making of the younger generations was directed by the authority of grandparents [2-6], yet, in the course of time, and due to rise in educational status of children and youth, the authority of grandparents over offspring and grandchildren declined. The present work tries to reflect a perspective of grandparents with special reference to their own lives, i.e. their safety and quality of life $[7,8]$.
Increasing life expectancy everywhere including Iran means that couples are likely to remain married for longer time; letting them appear as grandparents. Under such conditions the number of grandparents is ever increasing with especial reference to larger cities wherein more health and medical facilities are available. Years of living together contribute to mutual understanding and companionship to likely increase between old couples [9], or what is known in this paper as grandparents. By about age fifty, most couples have completed the task of raising children. From this age on, the couples gradually enter the period of grandparenthood. The remaining years of marriage - "the empty nest" brings a return to living with only one's spouse, and a decline in their socialization $[10,11]$.

However, more adults in midlife are facing challenges of caring for their aging parents or grandparents to their own children. Many families find that grandparents living to seventy and beyond require practical, emotional, and financial care that can be more complicated than raising their own children. Those born in 1950s - now in their sixties, will spend as many years looking after their aging parents, as they did caring for their own offspring [12-15]. 
Retirement also brings a change to family life. If the spouse has been a homemaker, the husband's retirement means that spouses will spend much more time together. Although the husband's presence is often a source of pleasure to both, more in the Western world, it sometimes undermines wives' established routines to the point of intrusion [16]. In case of Iran, wives not much welcome the retirement of husbands due to their ever presence at home, diminishing of family's income, and intervention of husbands in daily family routines etc.

The most difficult transition in married life comes with the death of a spouse. Wives typically outlive their husbands because of women's longer life expectancy and because wives are usually younger than husbands to begin with. Wives can therefore expect to spend a significant period of their lives as widows. Loneliness accompanying the death of a spouse is always difficult. This experience may be even harder for grandfathers or widows, who usually have fewer friends than grandmothers or widows do, and may be unskilled at housework [9].

In the present research, variables such as age, state of life, state of occupation, income, welfare, insurance, safety, education etc. have been investigated among the grandparents. They will be fully analyzed in a separate section. Due to better and improved supervision on children, their vaccination in infancy, and thereby their longer life expectancy and diminishing infant mortality rate (IMR) to as low as 5 within every 1000 live births in 2017 (WPDS 2017), boosting elderly and grandparents is highly expected; a scenario which widely affects the environment in general. This emerging phenomenon needs to be studied and analyzed in association with gerontology [17].

\section{Method of Research}

To do the research, the author first reviewed the necessary and background literature and theories on aging grandparents. In the theoretical section, relevant theories were searched, and the perspectives necessary were extracted, and used in the context. Similarly, in the empirical section as the backbone of the research, 452 questionnaires were administered through direct and face to face contacts with the respondents of 60 years of age and over, selected as random samples in various parts and neighborhoods of Tehran city. The selected grandparents were interviewed too, in the course of referrals. Eventually, the questionnaires were edited, electronically extracted and tabulated in the form of designed tables (Table 1).

Table 1: Multi-indicator Perspective of Grandparents in Tehran.

\begin{tabular}{|c|c|c|c|c|c|}
\hline \multicolumn{6}{|c|}{ The Way Grandparents are Approached in the Family } \\
\hline \multirow[t]{2}{*}{ Grandfathers } & $100 \%$ & With justice & With affection & With discrimination & \\
\hline & & $34.19 \%$ & $52.94 \%$ & $12.87 \%$ & \\
\hline Grandmothers & $100 \%$ & $16.42 \%$ & $68.66 \%$ & $14.93 \%$ & \\
\hline \multicolumn{6}{|c|}{ The Way the Ideas/Experiences of Grandparents are Used in the Family } \\
\hline \multirow[t]{2}{*}{ Grandfathers } & $100 \%$ & Use of ideas & Non-use of ideas & & \\
\hline & & $69.85 \%$ & $30.15 \%$ & & \\
\hline Grandmothers & $100 \%$ & $61.69 \%$ & $38.31 \%$ & & \\
\hline \multicolumn{6}{|c|}{ Safety and Psycho-Mental Dependency of Grandparents } \\
\hline \multirow[t]{2}{*}{ Grandfathers } & $100 \%$ & Visiting relatives & Visiting friends & Visiting offspring & None \\
\hline & & $26.47 \%$ & $10.66 \%$ & $49.26 \%$ & $13.60 \%$ \\
\hline Grandmothers & $100 \%$ & $27.61 \%$ & $6.47 \%$ & $59.45 \%$ & $6.47 \%$ \\
\hline \multicolumn{6}{|c|}{ Safety Dependency of Grandparents } \\
\hline \multirow[t]{2}{*}{ Grandfathers } & $100 \%$ & Adequate income & Care by offspring & Care by nurse & \\
\hline & & $42.65 \%$ & $55.88 \%$ & $1.47 \%$ & \\
\hline Grandmothers & $100 \%$ & $27.61 \%$ & $70.15 \%$ & $2.24 \%$ & \\
\hline \multicolumn{6}{|c|}{ Priority of Grandparents' Lives } \\
\hline \multirow[t]{2}{*}{ Grand fathers } & $100 \%$ & Living alone & Living in birth place & Living with spouse & Living with offspring \\
\hline & & $6.99 \%$ & $22.06 \%$ & $54.04 \%$ & $16.91 \%$ \\
\hline Grandmothers & $100 \%$ & $10.45 \%$ & $14.18 \%$ & $41.29 \%$ & $34.08 \%$ \\
\hline \multicolumn{6}{|c|}{ Grandfather Dead and State of Life of Grandmother } \\
\hline \multirow[t]{2}{*}{ Grandfathers } & $100 \%$ & Living alone & Living with offspring & & \\
\hline & & $72.78 \%$ & $27.22 \%$ & & \\
\hline Grandmothers & $100 \%$ & $60 \%$ & $40 \%$ & & \\
\hline
\end{tabular}




\section{Findings}

In referral to families to find out about different characteristics of grandparents, the researcher could find 272 alive grandfathers againt 402 alive grandmothers, i.e. a sex ratio of 68 males for every 100 females or grandmothers. Within those alive, $75 \%$ used to live together, while $25 \%$ of those elderly couples used to live with their offspring. In another query, the author came to know that $60 \%$ of sample grandfathers lived alone, while $40 \%$ used to live with their offspring. In this query, $73 \%$ of grandmothers used to live alone, while $27 \%$ used to live with their offspring. Similarly, occupational status of grandfathers was as such: $27 \%$ were busy in jobs, $41 \%$ pensioners, $1 \%$ had no pension at all, $7 \%$ were depended on offspring and finally $24 \%$ were depended on their own wealth In this regard, only $2.75 \%$ of grandmothers were engaged in jobs, $35.78 \%$ were pensioners, $15.6 \%$ had no pensions, $18.35 \%$ were depended on their offspring and $27.52 \%$ were depended on their own wealth.

Income being an important factor in old age, it was found that: $14 \%$ of grandfathers asserted to earn about 107 US \$ a month, $16.5 \%$ declared to have monthly income of about US $\$ 100$ and 160. Those having monthly income of US \$160 and 180 were $21 \%$, grandfathers with monthly income of US \$ 180 and 267 demonstrated $20.5 \%$, and finally $28 \%$ declared to have an income above US \$ 268 a month. So far as the grandmothers' income status is concerned, they declared their monthly income as such: $35.36 \%$ had income of less than US \$ 107 per month, 19\% of grandmothers had income of between US \$ 107 and 160, 15.22\% had monthly income of US \$ 160 and 180, 14.07\% had monthly income of US \$ 180 and 267 , and finally $16.35 \%$ of grandmothers declared to have a monthly income of US \$ 268 and above.

While insurance plays a determining role in the safety and security of the aging grandparents, $82.72 \%$ of our sample grandfathers declared to have insurance and the rest of $17.28 \%$ declared not to be under any health insurance coverage. In a query regarding the health insurance of grandmothers, $84 \%$ had insurance, and $16 \%$ did not have it [18].

In another table prepared to find out about the health conditions of grandfathers the data collected were as follows: $36.03 \%$ were healthy, $11.75 \%$ had arthritis, $20.22 \%$ had some heart disease, $7 \%$ had diabetes, and $25 \%$ had other diseases. In case of grandmothers, $26.62 \%$ were healthy, $28.61 \%$ had arthritis, $12.94 \%$ had some heart diseases, $12.19 \%$ had diabetes, and 19.65 had other diseases [19].

Older generations are not quite educated in Iran. Therefore, a perspective of the educational status of grandfathers could be reflected as follows: $26.84 \%$ uneducated, $30.88 \%$ had only primary education, $12.5 \%$ education of below ninth grade, $24.27 \%$ had finished secondary school or below, $1.84 \%$ had finished a diploma or college degree, $1.84 \%$ had a B.A./ B.Sc., and also $18.4 \%$ had a master's degree or above. Educational reflections on grandmothers is a bit different from those of grandfathers. Table concerning grandmothers states that $46.02 \%$ of them were illiterate, $30.6 \%$ had primary education, $6.47 \%$ had education of below ninth grade, $13.33 \%$ had finished secondary school or below, $2.24 \%$ had finished a diploma or a college degree, $1 \%$ had a B.A, B.Sc, and only $0.25 \%$ of the sample grandmothers had master's or above [20].

Classification of grandmothers according to their general feelings could be illustrated as such: $19.12 \%$ of our sample grandfathers declared to have loneliness feeling, $44.12 \%$ had impatience or bad mood feelings, $7.72 \%$ had feelings of nonassistance or helplessness, $12.5 \%$ feared death, and finally $16.54 \%$ of grandfathers declared no remarks. In case of grandmothers, feeling of loneliness among them was $30.35 \%$, impatience/bad mood was $30.6 \%$, feeling of non-assistance $14.43 \%$, fear of death was $12.19 \%$, and $12.44 \%$ declared no remarks [21].

Another table indicating grandfathers' status could be reflected as follows: $10.66 \%$ were in poverty, $29.41 \%$ were in social isolation, $30.15 \%$ suffered insecurity, and $29.78 \%$ gave no comments. Similarly, grandmother respondents were found, 9.2\% in poverty, $25.87 \%$ in isolation, $35.82 \%$ in insecurity, and $29.1 \%$ with no comments [22].

In a query regarding the satisfaction of grandfathers, various reflections were found accordingly, i.e. 59.19\% were satisfied with their lives, $18.75 \%$ were not satisfied with their lives, $8.82 \%$ expressed happiness, and finally $13.24 \%$ expressed grief. In case of grandmothers, $49.5 \%$ were satisfied with life, $16.17 \%$ were dissatisfied with their lives, $9.95 \%$ were happy, and $24.38 \%$ were grieved.

Similarly, attitudes of grandfathers towards different conditions were measured as such: $54.04 \%$ were satisfied with their marriage, $6.25 \%$ were discontented with their marriage, $5.15 \%$ were satisfied with their income, $10.66 \%$ were discontented with their income, $11.03 \%$ of the grandfathers were satisfied with the atmosphere/ their surroundings, and finally $12.87 \%$ of the grandfathers were discontented with their atmosphere around. In terms of grandmothers, the data showed that $35.82 \%$ were satisfied with their marriage, $10.7 \%$ were discontented with their marriage, $3.48 \%$ were satisfied with their income, and $15.17 \%$ were discontented with that. Similarly, $15.17 \%$ of grandmothers studied were satisfied with the atmosphere around them, and 19.65\% were dissatisfied with that. Other characteristics pertaining to the way of life of the grandparents and approaches towards them in the family are reflected in a compact table as follows.

\section{Discussion}

Grandparents can be of great help to their children. They can influence a family's adjustment, and often provide support to the entire family. They often serve many purposes of their grandchildren that their parents cannot fulfill. To be effective supporters, grandparents must first have their own needs and concerns responded and addressed. They have the potentiality of being each other's best resources. 
Grandparents not only in Iran, but in any other country could be the primary caregivers for millions of children, especially in modern time that many mothers work outside home. They could be increasingly of great help to the health and welfare of the "third generation". At the time when children are facing unprecedented stresses, and many parents are busy outside home, they could be a good reservoir of knowledge and parenting wisdom. Therefore, these great sources of advice and experience must well be protected.

To focus attention on the phenomenon, and provide grandparents with the recognition that they deserve, the present research has been started. Grandparents bridge between the last generation(s), and the new generation(s). They transfer old culture and values to the younger generation(s) through their caregiving to contribute to their social mobility. While they benefit their grandchildren, they are benefited by them too. Therefore, the youth must learn how to value grandparents in our society. Moreover, it must become a part of our culture, and globalized too. Similarly, grandparents can be updated more through contacts with grandchildren, and that prevents them from social exclusion.

To promote national quality of life, clean safe environment must be created, education and culture must be promoted economic opportunities should be provided at all levels, and finally the youngest and the oldest (grandparents) must be respected regardless of class, creed and race in a given society. However, environmentalism is a relatively new approach which argues that the physical world is being harmed and this will increasingly impact on our grandparents' welfare. However, sociologists argue how environmental problems are linked to particular cultural values, economic arrangements and welfare of aging grandparents [13].

Safety of grandparents depends on many factors such as providing social work and health services to them, and also improving their social, physical and mental well-being. Though grandparents are usually of the age of being retired, yet, they may be activated to maintain secondary functions such as giving care to their grandchildren in parent-absent homes, assisting each other (the two spouses), keeping their independence for longer time and so on. However, a century back or so, about half of children died before the age of twenty, and few could live to forty [17]. Under such conditions the chance of having grandparents was very low.

With increase in longevity and life expectancy, the number and proportion of grandparents are increasing more than ever before. Under such conditions, social security in old age is very vital to provide them with. The baby- boomers of mid 20th century are gradually turning to $60-65$, and adding to the number of grandparents in Iran. But, just as the physical problems of aging, intellectual and psychological changes accompany aging too. In short, we can state: "What goes up must come down" [3]. What they immediately need now is better income, more social security, and better coverage of health insurance. So, increase in old age means more grandparents. The trend is likely to increase up to 2020 due to high population growth rate of 1960s. However, as the number and proportion of the elderly people increase, the healthcare system will experience an unprecedented influx of grandparents with physical and mental health problems, i.e. various age-related issues will make their care more complex, and necessary.

Not all the elderly grandparents are in a state of safety, some minorities face challenges associated with addiction, mental health problems, Alzheimer's disease, dementia etc. Similarly, prevalence of depression among them is quite on increase in modern time. But, fortunately, as a result of advances in medical sciences, many old grandparents can be treated upon clinicians' diagnosis. That is largely possible in the industrial countries, and less practical in developing societies including Iran.

Recent studies demonstrate that many grandparents afflicted by depression, hypertension, diabetes, heart failure and many other ailments can be prevented, and in some cases treated, if means enough are available. What is more difficult with the elderly is mental disorders which cannot easily be cured. However, despite all the advances taken place at different rates, and in different societies, many physicians are unlikely to recognize and diagnose mental health problems in older adults in the early stages of Alzheimer's disease. Likewise, if and when diagnosed, most elder patients do not receive treatment. In case of physical problems, fractures etc., physicians and clinicians feel more responsible and pay more attention rather than mental problems and cases. So, in many cases, mental illness and aging problems which usually happen to grandparents are ignored in contemporary time and in many societies.

Factors such as the age of grandparents, and whether they both are alive - living together; all contribute to the quality of life of grandparents in Iran [23].

Similarly, occupational position of grandparents, their pensions, their "age and work" their, income and as a whole their economic position affect their quality of life, their health conditions etc. In Iran, not all the grandparents hold insurance. Therefore, those who have it, can practically have better and more immune life. Educational status of grandparents also narrows the gap between their grandchildren and them. Education has much played role in increasing life expectancy, or so to say, it has contributed to having more grandparents, and in the future the number and proportion will be much higher [12]. But, unfortunately not all the grandparents are educated. While many grandparents are in need of help and emotional supports of their children, or grandchildren, that often does not happen at the current complicated, socio-economic and cultural conditions.

\section{Theoretical Perspectives}

According to many economic demographers, an aging population and increase in the number of grandparents lead to negative consequences in terms of growth of output per capita. A decreasing ratio of the working-age population to the total population contributes to the increases of the ratio of dependents (grandparents) to working people and the active members of families. This phenomenon is increasingly appearing in the 
contemporary world with special reference to the countries with longer life expectancy.

The theory of optimal life-cycle human capital investment that has been developed by Bon-Porath (1967), Mincer [19] \& Becker [4], argues that rapid technological change in the form of education contributes to be almost exclusively concentrated at younger ages, poses challenges for rapidly aging population or grandparents. Hence, the relationship between age and human capital investment and consequently productivity growth cannot be seen in isolation from organizational and institutional factors. Under these circumstances the number of the grandparents is ever increasing, But, due to poor planning and controversial conditions, large number of aging grandparents are not in healthy and quality conditions in Iran.

No any economic theory provides a clear view as to how aging affects productivity. Thus, health limitations tend to reduce employment opportunities of aging grandparents. In response to lower earnings potential, older grandparents with health limitations are likely to reduce hours of work, and retire at earlier ages. Poor health also changes the grandparents' own assessment of the value of themselves. Yet, less is known about the relationship of grandparents' health and retirement in the rapidly aging countries of Asia including Iran.

Japan provides a good example of grandparents' health, retirement within them and so on. It has the most rapidly aging population (grandparents) in the world, and currently has the highest proportion of people age 65 and older. In 1980, only $9.1 \%$ of the population of that country was aged 65 and older. By 2004 this percentage had increased to $19.5 \%$, and current projections indicate that in 2025 , about $31 \%$ of the Japanese population will be aged 65 and older. That is, about one-third of population will be grandparents [20]. Hence, many other countries will have larger number and proportion of grandparents in the years to come.

Another perspective emphasizes the consequences of demographic change for long time economic growth. Anderson [1], for example, estimates the effect of population aging, and appearance of larger number of grandparents on average growth rate which is a down-ward trend. Bloom \& Williamson [11] add that the ratio of the non-working- age population (grandparents), to the working age population between 1965 and 1995; suggesting that a baby-boom generation would create a wavelike pattern of real GDP per capita over time. Bloom and Williamson believe that as baby-boomers increase the head count immediately after birth, they reduce per capita income, and the final incidence is on grandparents who are often pensioners.

It is well accepted that the future economic output in most industrialized countries must be achieved by a smaller and older labour force. A key question is how this development might affect labour productivity as measured by output per worker [10]. In the view of many economists, an aging population or increasing grandparents has negative consequences for growth in output per capita. Therefore, countries with such population structures must search alternatives to respond to the shortage of their human labor force (15-64). However, though the number of grandparents is increasing, their economic conditions are at risk in countries like Iran. They are at present highly dependent on their offspring, and the scenario will be worse in the years to come.

Until recently grandparenthood has been a neglected area of study (Cunningham-Burley 1986). Academic interest has been much more widespread in the USA [6]. Roles that have been identified are surrogate parent [24]. One matter of concern has been the lack of legal rights and obligations that parents have in relation to their grandchildren.

Upon the studies done, it was found out that grandmother was the second most frequent source of child care for women in employment [18]. In that, large number of pre-school children of working mothers use grandmothers to look after these children during the absent-time of mothers. Almost the same amount of help and care are offered to school-aged children by grandmothers. However, this is a sort of exchange, i.e. while the daughter sees her visits as "keeping an eye on mum", mum may see the visits as the daughter turning to her for help and advice [14]. He stresses that to be on the receiving end in old age amounts to an abrupt reversal of the parental role.

Older people being the whole of a generation survived to a certain age, is the result of demographic changes in the population, i.e. the outcomes of longer life expectancies, lower birth rates, and an older average age of giving birth [25]. This scenario eventually leads to the phenomenon of grandparents. It has been recognized that such changes are likely to result are significant increases in elder abuse which includes grandparents too. Sociologically speaking, disproportionate increases in the number of dependent elders (Grandparents) relative to working-age individuals may result in higher stress levels among caregivers and increase abuse opportunities, and thus may act to increase the rates of grandparents abuse.

Findings, and the literature on elder mistreatment, and misbehavior with the elderly appears to emphasize dependency and stress as two of the most significant factors. For example, a positive relationship between abuse and stressful workplace environment has been emphasized in Pillemer \& Finkelhor [22], and Pillemer \& Moor [21]. In many cases, the elder and grandparents' abuses are hidden, and as a normal and routine movement - depending on societies and cultures, poverty and affluence etc. For example, Harrington et al. [14] found a positive relationship between nurse staffing hours and nursing home deficiencies. The specific mechanisms that lead to increased levels of abuse, neglect, and exploitation, are not clear, what is consistent among the studies is that, a decrease in the qualified workforce is inversely related to abuse.

However, safety and quality of life of grandparents highly depend on health status, personality traits, and ethno-cultural backgrounds of those around such elderly people. Therefore, such people may abuse the elderly, or have good behavior with them. 
In the present paper, we are unable to uncover all the dimensions regarding the grandparents' lives because of data limitations.

\section{Conclusion}

Figures indicate that the aging people or so to say, grandparents are increasing in Iran. Personal characteristics of the elderly such as health status, personality traits, personal problems, and socioeconomic backgrounds of grandparents are different in this paper. Therefore, grandparents represent different reflections. Similarly, different educational status, income conditions, work and health conditions of these elderly people prior to their retirement have given them different quality of life in their old age as grandparents. Increase of grandparents in Iran to about seven times during sixty years (1956-2016) means that the elderly's needs have increased at higher proportions, and any failures or neglects towards that, will be elder abuse within them. In terms of norms and culture, the older grandparents rely on their children, and in that, grandmothers more rely on their daughters. Similarly, as residing in nursing homes is not much common in Iran, the only alternative left for these people, is to be supported by their offspring and younger family members. In this paper we are unable to measure the quality of life of the elders any further due to resource and data limitations, which should be addressed in future studies.

Moreover, due to current young population structure in Iran, the country will face much higher number and proportion of elderly grandparents by 2030 . As the average life expectancy has also reached 75.5 years in 2016 [26], we must logically expect more grandparents, especially women. However, issues such as social stigma and low status, employment and compulsory retirement, financial services and insurance, transport and medical treatment are highly problematic for the elder grandparents less in Tehran and more in Iran as a whole [27].

\section{References}

1. Anderson B (2001) Scandinavian evidence on growth and age structure. Regional studies 35(5): 377-390.

2. Asefi A (1973) Today's grandfathers and grandmothers. Anjoman Oliya va Morabian Publications, Teheran, Iran, p. 16.

3. Baltes PB, Shaie KW (1974) The myth of twilight years. Psychology Today 7(10): 35-39.

4. Becker G (1964) Human capital, University of Chicago Press, Chicago, USA.

5. Becker GS (2004) Economic approaches to understanding families. Understanding and Optimizing Human Development: From Cells to Patients to Population 1038 (1): 201-205.
6. Bengstone V, Robertson J (1985) Grandparenthood, Sage Publications, Beverly Hills, California, USA.

7. Ben Porath Y (1967) The production of human capital and the life cycle of earning. Journal of Political Economy 75(4): 352-365.

8. Benoit HM (2008) Lecture delivered at the centre for addiction and mental health. University of Toronto, Canada.

9. Berado FM (1970) Survivorship and social isolation: The case of the widower. The Family Coordinator 19: 11-25.

10. Blanchet D (1992) Does an aging labour force call for large adjustment in training or wage policies? In: Johnson P, Zimmermann KF (Eds.), Labour market in an aging Europe. Cambridge University Press, USA.

11. Bloom DE, Williamson JG (1998) Demographic transitions and economic miracles in emerging asia. World Bank Economic Review 12: 419-455.

12. Blundell J (2001) Active sociology, Essex, Longman.

13. Cylke FK (1993) The Environment, Harper Collins, New York, USA.

14. Harris C (1969) The Family, Allen and Unwin, London.

15. Jarrett, Robin L (1994) Living poor: family life among single parent african american women. Social Problems 41(1): 30-49.

16. Kalish R (1982) Late adulthood: Perspectives on human development $\left(2^{\text {nd }} \mathrm{edn}\right)$, Brooks, Monterey, California, USA.

17. Lenski G, Lenski J (1995) Human societies: an introduction to macrosociology, ( $7^{\text {th }}$ edn), McGraw-Hill, New York, USA.

18. Martin J, Roberts J (1984) Women and employment: a lifetime perspective, HMSO, London.

19. Mince J (1974) Schooling, experience, and Earnings, Columbia University Press, New York, USA.

20. Ogawa N (2003) Population aging and its impact on the socio-economic system in Japan, Aging in Japan 2003, Japan Aging Research Centre, Tokyo, Japan.

21. Pillemer KA, Moore DW (1989) Abuse of patients in nursing homes: Findings from a survey of staff. Gerontologist 29(3): 314-320.

22. Pillemer KA, Finkelhor D (1989) Causes of elder abuse-caregiver stress vs problem relatives. American Journal of Orthopsychiatry 59(2): 179187.

23. Results of the Decennial population and housing censuses in iran 19562006

24. Victor C (1994) Old Age in modern society. Chapman and Hall, London.

25. Voelker R (2002) Elder abuse and neglect, a new research topic. Journal of American Medical Association 288(18): 2254-2256.

26. World Population Data Sheet (2016) PRB, Washington DC, USA.

27. World Population Data Sheet (2017) PRB, Washington DC, USA. 

(c) Creative Commons Attribution 4.0

For possible submissions Click Here

Submit Article

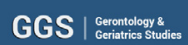
Benefits of Publishing with us

- High-level peer review and editorial services

- Freely accessible online immediately upon publication

- Authors retain the copyright to their work

- Licensing it under a Creative Commons license

- Visibility through different online platforms 